\title{
Effects of Tai Chi and Qigong in Children and Adolescents: A Systematic Review of Trials
}

\author{
Jody L. Riskowski ${ }^{1,2} \cdot$ Rania Almeheyawi $^{1}$
}

Received: 30 June 2017 / Accepted: 18 August 2017 / Published online: 14 September 2017

(C) The Author(s) 2017. This article is an open access publication

\begin{abstract}
Tai Chi and Qigong are meditative movementbased practices that necessitate sustained focus, precision in movement and strictness of action. Due to the requisite high levels of concentration, Tai Chi and Qigong have mainly been studied in adult populations, where systematic reviews have shown positive efficacy. As facilitators and barriers to Tai Chi and Qigong practice in youth are different relative to adults, efficacy of the practices may also differ. Therefore, this systematic review investigates and evaluates intervention studies of Tai Chi and/or Qigong in adolescent populations (18 years and under) to determine how these interventions have been applied as well as synthesize intervention outcomes. For the 13 studies (16 manuscripts) included in the work, frequency and duration of the Tai Chi or Qigong intervention ranged from a two sessions per week for 5 week to five sessions per week over a year. Synthesis of results showed there were two outcome domains: (1) psychological wellbeing and behavior, and (2) physical health and function. Results of the synthesized data suggest that Tai Chi or Qigong may positively improve physical health and function, while its effect on psychological wellbeing and behavior are inconclusive with most studies reporting improvements or no effect.
\end{abstract}

Jody L. Riskowski

Jody.Riskowski@gcu.ac.uk

Rania Almeheyawi

Rania.Almeheyawi@gcu.ac.uk

1 Institute for Allied Health Research, Glasgow Caledonian University, 70 Cowcaddens Road, Glasgow, UK

2 Department of Psychology, Social Work and Allied Health Sciences, Glasgow Caledonian University, Glasgow, UK
Keywords Complementary and alternative medicine . Mind-body · Teenage, Juvenile

\section{Introduction}

Approximately $40 \%$ of adults in the United States use complementary and alternative medicine (Harris et al. 2012), with approximately $25 \%$ of these individuals taking part in mind-body activities (Chacko et al. 2014). What is now the United States National Center for Complementary and Integrative Health (NCCIH) defines these mind-body activities as practices and interventions that intended to promote the connection of the mind to help heal itself through modifying biological, physiological and/or psychosocial processes (Straus 2000). Thus, mind-body activities are noted as therapeutic practices that "focus on the interactions among the brain, mind, body, and behavior, with the intent to use the mind to affect physical functioning and promote overall health" (Elkins et al. 2010, p. 128). The healing properties of mind-body practices is based on the belief of what a person thinks, what a person feels, and how a person moves can influence health and healing (Chen et al. 2013). The importance and connection between the mind, emotion, and behavior in health lays at the heart of many complementary and alternative medicine or traditional medicines, such as Native American, Chinese, Tibetan, Greek, and Ayurvedic medicine, among others (Ben-Arye et al. 2006).

Categorizing within the mind-body therapies are meditative movement-based activities, such as Tai Chi and Qigong (Larkey et al. 2009), whereby the attention in the activity is on the movement, breath and mind. The Tai Chi and Qigong known today developed from ancient Chinese martial arts that combined deep diaphragmatic breathing with basic postures that purposefully move from one to another. The 
practice of Tai Chi and Qigong can range from dynamic, powerful actions to gentler, continuous movement patterns, with all forms having an attention to exact posturing and body positioning. As Tai Chi and Qigong share theoretical roots and linkages to traditional Chinese medicine, as often these two meditative movement activities are considered equivalent interventions (Jahnke et al. 2010).

Uptake in practices such as Tai Chi and Qigong have steadily risen over the years (Clarke et al. 2015), particularly among middle aged and older adult populations (Wang et al. 2004). Alongside the rise in people practicing these activities, is the rise in research and interventions evaluating outcomes associated with Tai Chi or Qigong (Yang et al. 2015). Intervention studies of Tai Chi or Qigong in adults have focused on specific patient populations, such as those with musculoskeletal conditions such as knee osteoarthritis (Chenchen Wang et al. 2009), patients with diabetes (Cavegn and Riskowski 2015), patients with Parkinson's Disease(Hackney and Earhart 2008) as well as general populations of middle-aged adults(Goon et al. 2009; Thornton et al. 2004) and older adults (Fuzhong et al. 2001; Wolfson et al. 1996). These intervention studies of the mind-body practices of Tai Chi and Qigong are research studies whereby participants are allocated to an intervention in order for researchers to evaluate its effects on health-related outcomes and wellbeing. Usually intervention studies have two or more treatment arms (e.g., Tai Chi, Qigong, no intervention), and the intervention occurs over a set number of weeks or month. Within the intervention study, baseline and posttesting, which is testing before and after the intervention (e.g., Tai Chi, no intervention) respectively, typically occurs. The measures from pre- and post-post intervention allow the researcher a means to evaluate the participants' changes in health and wellbeing through the study and between the intervention arms [e.g., Tai Chi or Qigong versus its comparator (e.g., no intervention)]. However, other studies also simply track health-and wellbeing- related changes over time given an intervention (e.g., Tai Chi, Qigong) without having a comparator arm, and this is known as a single-arm intervention trial.

When researchers evaluate the totality of the evidence around a single intervention, such as in a systematic review, it can help clinicians, health-care workers, and the general population make informed decisions as to whether an intervention is beneficial or not to the populations that have been studied. If specific populations have not been studied with respect to the intervention, researchers may make the assumption that because it helped one population it could help another. This assumption of two different populations yielding similar outcomes given a similar intervention may or may not be a correct assumption, such as the case in using Tai Chi to improve cardiovascular fitness, where it has been shown to be helpful in older adults (Lan et al. 1998) but not in young adults (Zhuo et al. 1984). One reason given for the lack of cross-over of effect between change in cardiovascular fitness between young and older adults may be that the increase in physical activity that arises with the Tai Chi or Qigong intervention for a sedentary older adult is relatively greater than the increase in physical activity that a sedentary young adult may experience (Zhuo et al. 1984). Thus, the developmental stage of when Tai Chi or Qigong interventions are commenced may affect the outcomes of the health- and wellbeing-related outcomes, casting doubt on the assumption that efficacy between youth and adult population is similar.

Most research and intervention studies evaluating the health- and wellbeing related effects of Tai Chi or Qigong have been in middle and older adult populations. Tai Chi and Qigong interventions studies and systematic reviews, which seek to evaluate the totality of evidence of the intervention studies, have shown these practices to reduce pain (Kang et al. 2011; Wang et al. 2004), increase range of motion (Liu and Frank 2010; Ye et al. 2014), enhance proprioception (Liu and Frank 2010), improve mental health and wellbeing (Chenchen Wang et al. 2010, 2004), and boost cardiovascular and respiratory function (Niu et al. 2014), with few adverse side-effects (Yan et al. 2013). Correspondingly, qualitative studies in middle age and older adult populations report that Tai Chi practitioners partake in the activity due to the perceived or actualized ability of the practice e to help adults maintain and/or improve mental and physical health when living with chronic conditions (Uhlig et al. 2010) and with the aging process (Gryffin et al. 2015).

As studies suggest that the outcomes achieved in adult populations given a Tai Chi or Qigong intervention may not correspond to similar outcomes in youth or adolescent populations, there is a need to further evaluate the effects of these practices in populations under age 18 years of age. Part of the resistance to evaluating Tai Chi and Qigong in younger population may be that because these practices necessitate "sustained concentration, precision, [and] mechanical exactness", (Wall 2005, p. 230) there may not be the interest youth and adolescent populations to the degree that more active martial arts (e.g., Kung Fu, karate) may be able to sustain (Wall 2005). A qualitative study of Tai Chi or Qigong in an adolescent population, a sample of in-patients of a psychiatric department who had Anorexia Nervosa, noted that the slowness of activities was a barrier to appreciating the practice (Gueguen et al. 2017). These patients also struggled with the perceived mental and physical health benefits of the practice, with some noting positive gains for the regular practice while others struggled to find benefits. Regular and on-going practice is necessary for change through mind-body interventions of Tai Chi and Qigong (Wu 2002), so if youth and adolescent populations struggle to practice these activity, the benefits may not be realized. 


\section{The Current Study}

Although nearly $12 \%$ of US children and adolescents use complementary and alternative medicine (Barnes et al. 2008), often these complementary and alternative medicine and mind-body interventions for children and adolescent populations are based on the health and wellbeing outcomes noted in adult populations. As Tai Chi and Qigong necessitate sustained focus, precision in movement and strictness of action, it may (Lee et al. 2013) or may not (Wall 2005) appeal to young children and adolescents. However, it may also be that these elements-focused mind, awareness of body, and exactness of action-make Tai Chi or Qigong an appropriate intervention for children and teenagers (Lee et al. 2013). Therefore, the purpose of this systematic review is to evaluate use, effects, and reporting and methodological quality of peer-reviewed publication of Tai Chi and Qigong intervention studies in child and adolescent populations as well as to offer recommendations for future research. In the context of this systematic review, the intervention studies will be prospective, longitudinal research studies that included pre- and post-assessments of the participants who took part in more than one session of Tai Chi or Qigong, making it a longitudinal study. Ultimately, the goal of this systematic review is to determine how Tai Chi or Qigong intervention studies are implemented when they are used with youth population as well as to determine what the overall outcomes of the studies are.

\section{Methods}

This systematic review was submitted and approved through the PROSPERO registry of systematic reviews (CRD42015023217) and followed the Preferred Reporting Items for Systematic Reviews and Meta-Analyses (PRISMA) guidelines (Moher et al. 2009).

\section{Search Strategy}

The literature search was conducted on publication platforms of PubMed, Medline, Cumulative Index to Nursing and Allied Health Literature (CINAHL), Physiotherapy Evidence Database (PEDro), PsychINFO, and BioMed Central Ltd as well as controlled trial databases of Current Controlled Trials and ClinicalTrials.gov. Key words for the literature search included: Tai Chi, Taiji or Qigong, children, adolescent or youth, and random* (e.g., randomization, randomised), trial, study, or intervention. There was no explicit search of grey literature (e.g., unpublished, nonpeer reviewed document or government, academics, business and industry papers). The search was conducted on June 1, 2017 without language restrictions. Hand-searching of reference lists of articles meeting inclusion criteria was also conducted.

\section{Selection Criteria for Inclusion}

As the relative number of Tai Chi or Qigong studies in children and adolescents was assumed to be small, the inclusion criteria were purposefully designed to be inclusive. The inclusion criteria for this study were any movementbased Tai Chi or Qigong intervention trial published in peerreviewed or refereed journals with participants who were, on average, younger than 18 years of age. Studies had to have at least two or more session of Tai Chi as a part of the intervention and have a quantitative element of the research design. Studies of massage or meditation only were not included.

From the keyword and MESH (medical subject headings) terms search, duplicate manuscripts from the different databases were eliminated, with the remaining manuscripts passing into the title stage. In the title stage, manuscripts were eliminated if it was evident from the title that criteria regarding the methodology or population were not satisfied, with remaining manuscripts moving to the abstract stage. At the abstract stage, two reviewers independently reviewed abstracts of said publications to determine if inclusion criteria were met. Following the abstract stage, full-texts of the manuscripts were obtained and reviewed for inclusion based on the inclusion criteria of age of population and number of Tai Chi or Qigong sessions. Manuscripts written in languages other than English were included, and they were reviewed by others comfortable with the language of the particular text. If data from the same study were reported in multiple publications, these were reported as one study.

\section{Data Extraction}

From the included manuscripts, manuscripts were first reviewed to determine if they were of the same study population and collated as appropriate. After a data extraction pilot to ensure completeness of necessary information (Levac et al. 2010), data from included manuscripts were independently extracted and discussed subsequently where disagreements arose. Extracted data included year and study setting, sample size by gender, age, intervention duration and frequency, completion, retention and adherence, as well as study outcomes and findings.

\section{Evaluation of Methodological Quality}

Methodological quality of each manuscript was assessed using the dichotomized scoring system developed by the Critical Appraisal Skills Programme (CASP) (2007) (0 for not reported, unclear or not appropriate, 1 for reported and appropriate) and is fully reported (Faggion Jr 2016). The 
CASP tool was chosen as it is a widely used and well-developed critical appraisal tool to assess the methodological quality of studies (Sanderson et al. 2007). The same reviewers independently assessed each manuscript using this tool, with disagreements resolved through discussion until a consensus was met. Each manuscript was rated on a scale of 1 (poor quality) to 9 (high quality) based on the CASP criteria. Included manuscripts were considered as being high quality if they scored seven or more out of the nine items, near the $80 \%$ quality cut-point (Slavin 1995), with those scoring six or less being considered of low quality.

\section{Data Synthesis}

As the outcomes were not known a priori, all outcomes of the manuscripts were reported and summarized as either showing an effect (positive or negative) or no effect based on the reported information in the manuscripts. The final results were synthesized though the interpretive review, which incorporates the concepts identified in the studies into higher-order domains in order to provide a more structured approach to combining studies that may not have common measures or outcome tools (Evans 2002). The final domains and the links to these domains were evaluated by an independent consultant of this review.

\section{Results}

The initial keyword search yielded 5625 manuscripts. Through the systematic review of the titles, abstracts and full-text of the manuscripts, a total of 16 manuscripts were included, of which 13 were unique studies (Fig. 1). There were no completed trials noted on the controlled trial database that were not included in the final included studies.

The included studies were from Europe (Sweden, Germany, and Portugal), North (Canada and United States) and South (Argentina) American, Asia (mainland China, Taiwan, Hong Kong) and Australia. Combined, these studies included 696 participants (Table 1), with 360 youths participating in the Tai Chi or Qigong intervention arm and 336 in the comparator arm. Although one study did not report gender of participants (Bao and Jin 2015), approximately $54 \%$ of the included participant were girls. Mean age of the participants in these studies ranged from 8 (Lozada et al. 2014) to 14.8 (Bao and Jin 2015) years.

Target sample populations were varied, with three studies were targeting the general student body (Bao and Jin 2015; Lee et al. 2013; Witt et al. 2005), one targeting musicians (Sousa et al. 2012), and five targeting youth population with specific conditions, including those who had learning disabilities and behavioral problems (Baron and Faubert 2005), attention deficit hyperactivity disorder (ADHD)
Fig. 1 Flow chart diagramming the systematic review of articles to include in analysis

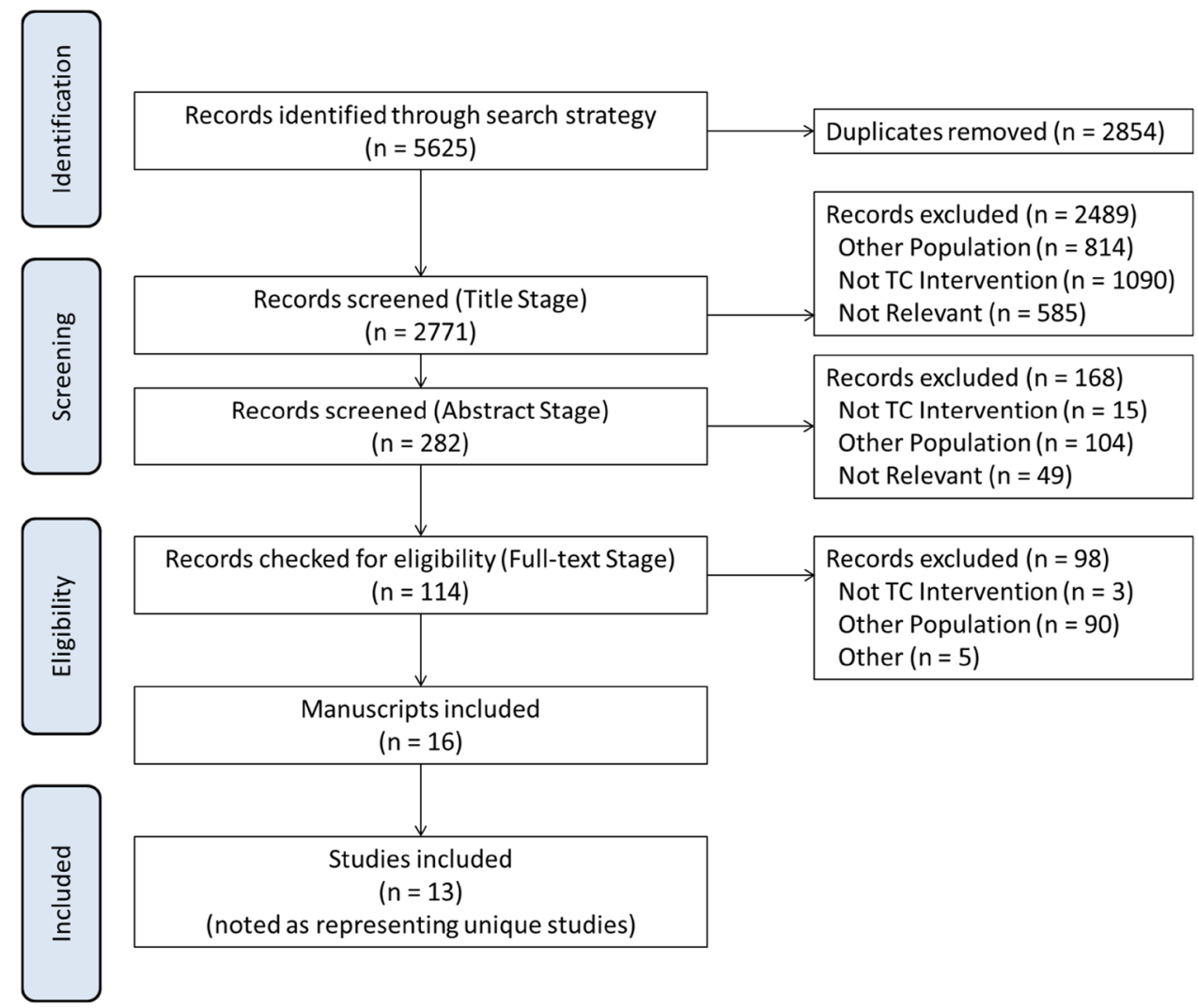




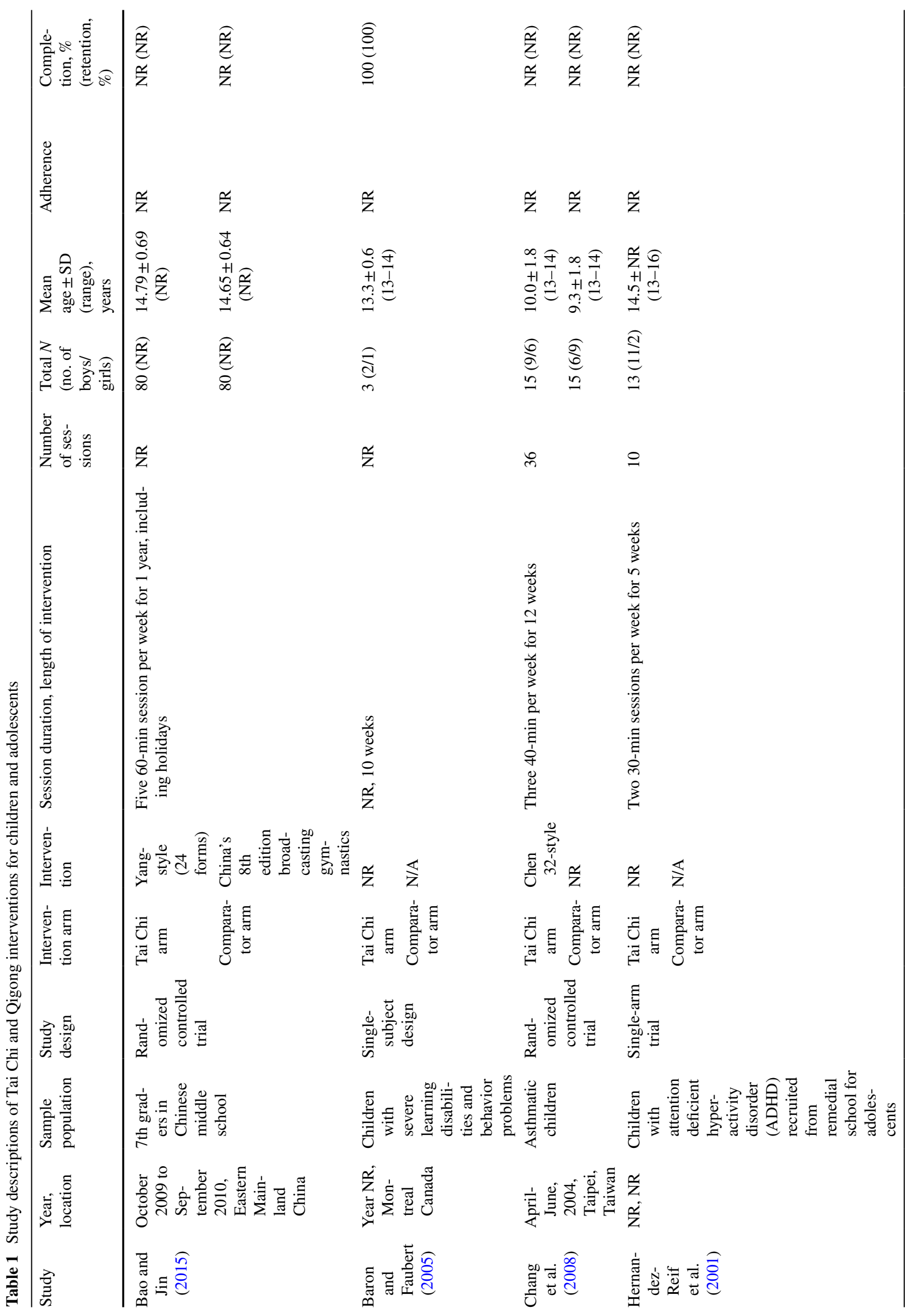




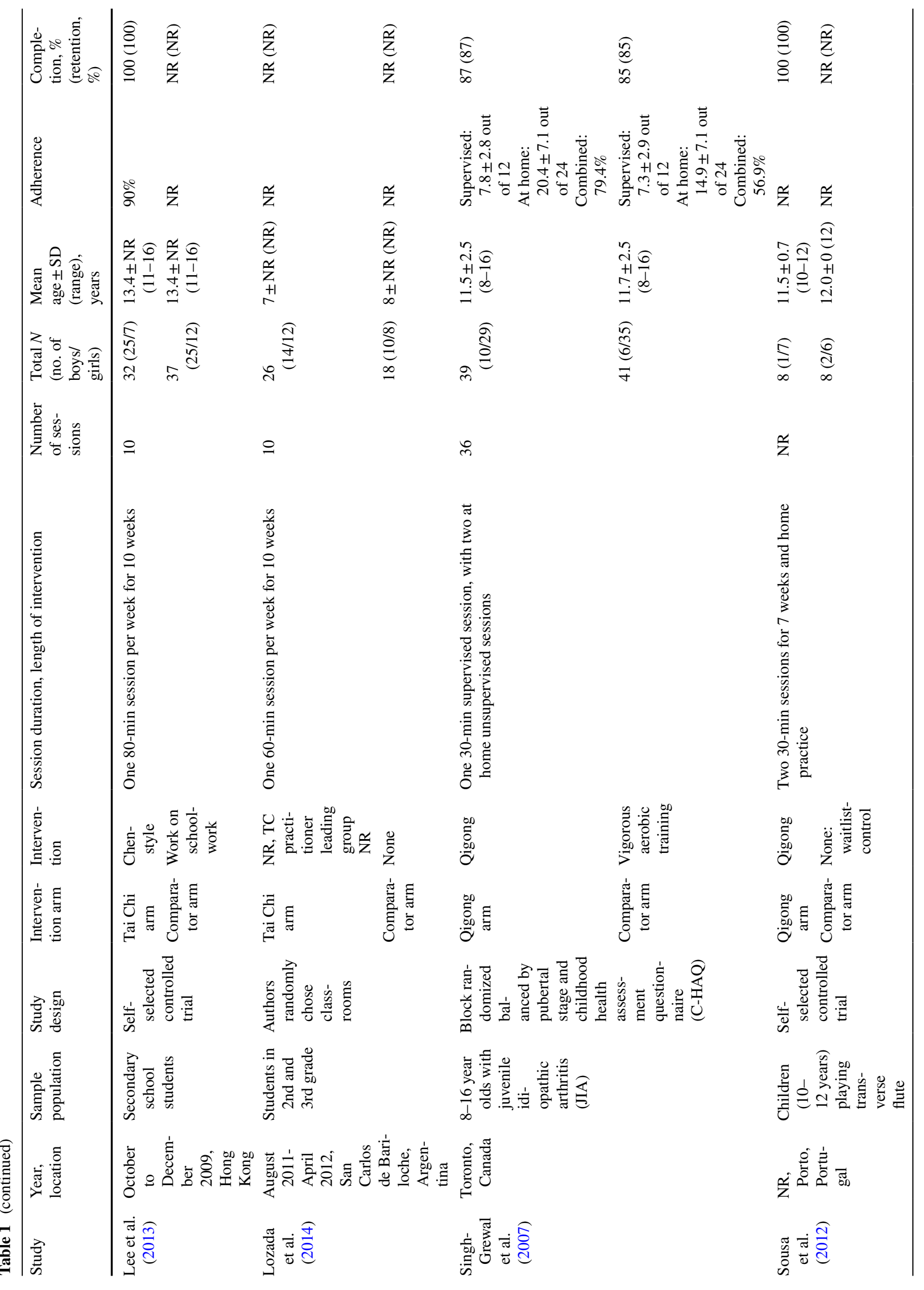




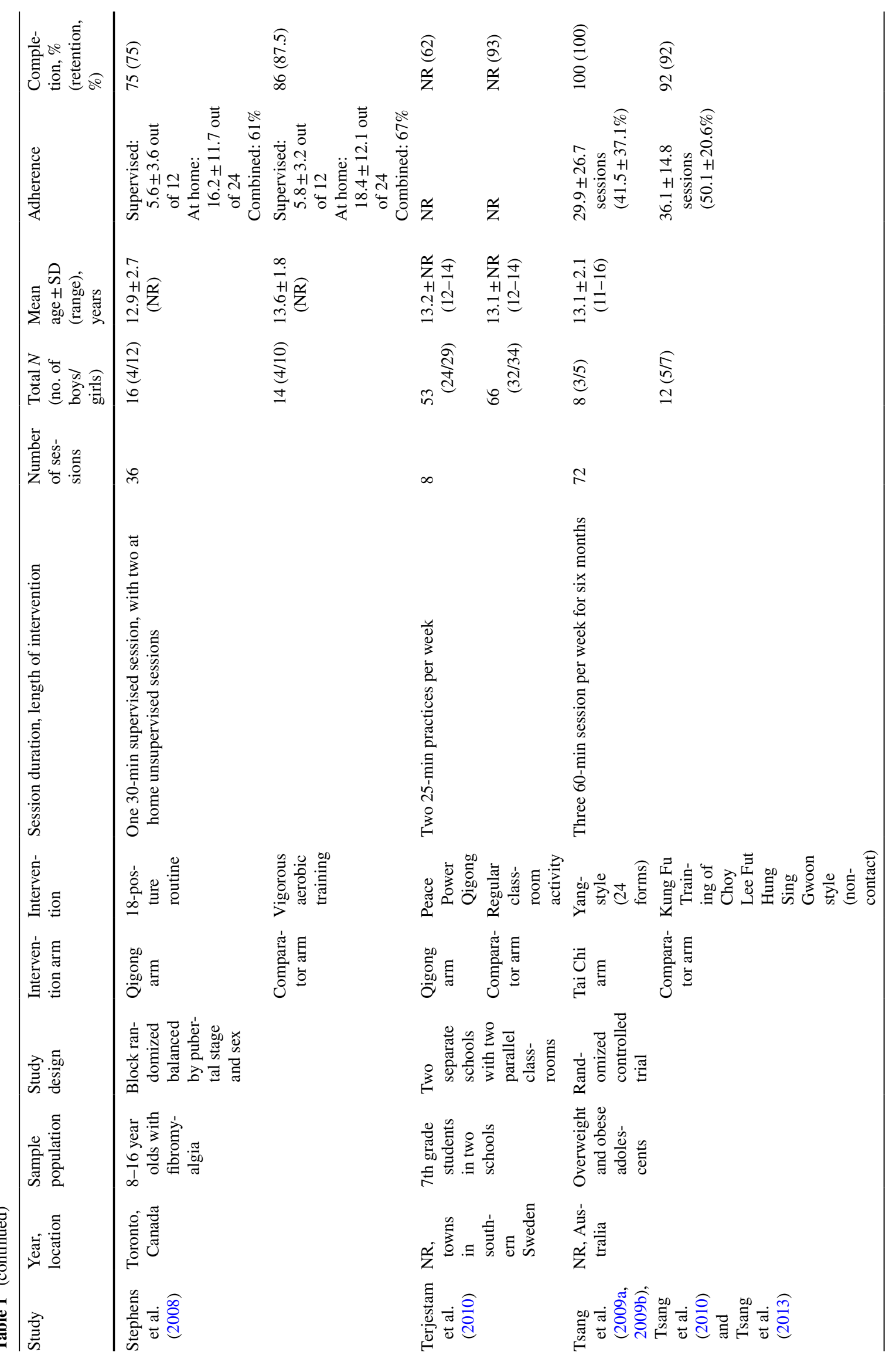




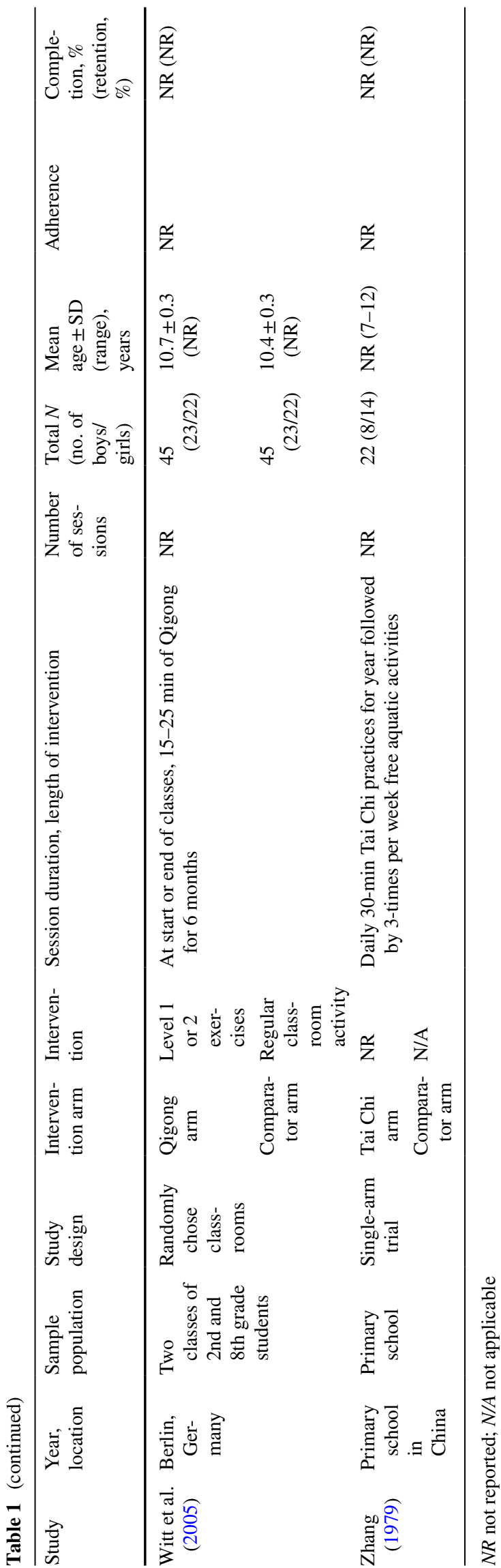

(Hernandez-Reif et al. 2001), juvenile idiopathic arthritis (Singh-Grewal et al. 2007), or fibromyalgia(Stephens et al. 2008), or who were overweight or obese (Tsang et al. 2009a, b, 2010, 2013).

In the Tai Chi or Qigong arm, nine studies did not report adherence, while four did, with adherence ranging from 42 to $90 \%$. Similarly, most studies did not report retention $(\mathrm{N}=6)$ or completion $(\mathrm{N}=7)$ rates. Of the studies that reported retention, four reported $100 \%$ retention $\mathrm{n}$ (Baron and Faubert 2005; Lee et al. 2013; Sousa et al. 2012; Tsang et al. 2009a, b, 2010, 2013), while the remaining three studies reported a retention rate of $62 \%$ (Terjestam et al. 2010), 75\% (Stephens et al. 2008), or 87\% (Singh-Grewal et al. 2007). Of the studies with reported completion rates, four reported 100\% completion (Baron and Faubert 2005; Lee et al. 2013; Sousa et al. 2012; Tsang et al. 2009a, b, 2010, 2013) while one reported 87\% (Singh-Grewal et al. 2007) and another $75 \%$ (Stephens et al. 2008). Adverse events were reported for two studies, with none reported in the Tai Chi or Qigong arm (Stephens et al. 2008; Tsang et al. 2009a, b, 2010, 2013), while Tsang noted adverse events in the comparator arm (Kung Fu) (Tsang et al. 2009a, b, 2010, 2013).

\section{Tai Chi or Qigong Intervention}

There was significant heterogeneity in the type of Tai Chi or Qigong practiced in the intervention (Table 2). Two studies reported using Yang-style (24 forms) Tai Chi (Bao and Jin 2015; Tsang et al. 2009a, b, 2010, 2013) or Chenstyle(Chang et al. 2011; Lee et al. 2013), with five having Qigong intervention (Singh-Grewal et al. 2007; Sousa et al. 2012; Stephens et al. 2008; Terjestam et al. 2010; Witt et al. 2005).

The included studies varied in allocation to intervention arm as well as frequency and duration of the Tai Chi or Qigong intervention. Five of the included studies were noted as randomized controlled trials (Bao and Jin 2015; Chang et al. 2008; Singh-Grewal et al. 2007; Stephens et al. 2008; Tsang et al. 2009a, b, 2010, 2013), two allowed for selfselection of trial arm (Lee et al. 2013; Sousa et al. 2012), and three were in schools were the study coordinators or schools choose the allocation of treatment to the classroom (Lozada et al. 2014; Terjestam et al. 2010; Witt et al. 2005). Two studies were single-arm trials (Baron and Faubert 2005; Hernandez-Reif et al. 2001) with no comparator. Of those with a comparator arm, four studies had an active comparator arm (Kung Fu (Tsang et al. 2009a, b, 2010, 2013), China's 8th edition broadcasting gymnastics (Bao and Jin 2015), and vigorous aerobic training (Singh-Grewal et al. 2007; Stephens et al. 2008)), while three had routine classroom activity (Lozada et al. 2014; Terjestam et al. 2010; Witt et al. 2005) or an additional schoolwork study period (Lee et al. 2013). One study had a waitlist control arm (Sousa et al. 
Table 2 Descriptions of practice sessions

\begin{tabular}{|c|c|}
\hline Study name & Typical Tai Chi session \\
\hline Bao and Jin (2015) & $\begin{array}{l}\text { During sessions, the martial arts coach demonstrated the Tai Chi movements, and participants imitated } \\
\text { motions and posture. New Tai Chi movements were introduces each session, and the entire sequence took } \\
10 \text { min to perform }\end{array}$ \\
\hline Baron and Faubert (2005) & A professional Tai Chi instructor taught the course. No further information regarding structure reported \\
\hline Chang et al. (2008) & Chen 32-style style practice \\
\hline Hernandez-Reif et al. (2001) & $\begin{array}{l}\text { Each session opened with slow raising and lowering of the arms synchronized to the breath for } 5 \text { min. They } \\
\text { were then taught to perform slow turning and twisting movements of the arms and legs, shifting the body } \\
\text { weight from one leg to the other, rotating from side-to-side and changing direction to the Tai Chi sequence } \\
\text { form }\end{array}$ \\
\hline Lee et al. (2013) & $\begin{array}{l}\text { A qualified Tai Chi instructor with more than } 10 \text { years' experience facilitated the sessions. In each session, } \\
\text { there was a 15-min warm-up phase, followed by a } 60 \text {-min activity phase of Chen-style Tai Chi and ending } \\
\text { with a 5-min cool-down phase. The Chen-style Tai Chi phase included body and truck rotation, hip and } \\
\text { knee flexion, weight shifting and reciprocal arm movements and balance, with the participants }\end{array}$ \\
\hline Lozada et al. (2014) & $\begin{array}{l}\text { Tai Chi intervention had four elements: (1) breathing, (2) Tai Chi practices, and (3) cooperative games, and } \\
\text { (4) reflective moment sitting in a circle. During guided breathing technique children kept eyes closed and } \\
\text { concentrated on each breath. Tai Chi practice was slow, flowing movements with changes of posture and } \\
\text { synchronization of movements. During cooperative games children played games that required working } \\
\text { together to achieve goals }\end{array}$ \\
\hline Singh-Grewal et al. (2007) & $\begin{array}{l}\text { Qigong based on Tai Chi forms and utilized 18-posture program, with each posture repeated } 8 \text { times. At end } \\
\text { of session was gentle stretching. Throughout session heart rate was less than } 75 \% \text { of maximum heart rate } \\
\text { (MHR), with MHR defined through } \mathrm{VO}_{2 \text { peak }} \text { protocol. Instructor student ratio was } 1: 4\end{array}$ \\
\hline Sousa et al. (2012) & Not reported \\
\hline Stephens et al. (2008) & $\begin{array}{l}\text { Qigong based on Tai Chi forms and utilized 18-posture program, with each posture repeated } 8 \text { times. At end } \\
\text { of session was gentle stretching. Throughout session heart rate was less than } 75 \% \text { of maximum heart rate } \\
\text { (MHR), with MHR defined through } \mathrm{VO}_{2 \text { peak }} \text { protocol. Instructor student ratio was } 1: 4\end{array}$ \\
\hline Terjestam et al. (2010) & $\begin{array}{l}\text { Twenty-five minute practice was on Peace Power Qigong, meditative movement experiences (few minutes of } \\
\text { relaxation, five sets of movements and self-massage) }\end{array}$ \\
\hline $\begin{array}{l}\text { Tsang et al. (2009a, 2009b); } \\
\text { Tsang et al. (2010); Tsang et al. } \\
\text { (2013) }\end{array}$ & $\begin{array}{l}\text { Yang-style Tai Chi sessions were led by a group of Tai Chi professionals. Sessions began with a warm-up } \\
\text { period of exercises, which lead into the Tai Chi practice. There was a short (1-2 min) break during the mid- } \\
\text { dle. During the session, Chinese-style music played in the background }\end{array}$ \\
\hline Witt et al. (2005) & $\begin{array}{l}\text { Level } 1 \text { and } 2 \text { exercises. Level } 1 \text { being } 15 \text { different exercises of the upper body repeated } 20 \text { times, and level } 2 \\
\text { including upper and lower parts of the body with background music playing }\end{array}$ \\
\hline Zhang (1979) & Not reported \\
\hline
\end{tabular}

2012). Supervised Tai Chi or Qigong intervention ranged from weekly a practice of $50 \mathrm{~min}$ (two $25 \mathrm{~min}$ sessions (Stephens et al. 2008)) to $5 \mathrm{~h}$ [five 60-min sessions (Bao and Jin 2015)]. Three studies requested participants undertake unsupervised home practice between the supervised sessions (Singh-Grewal et al. 2007; Sousa et al. 2012; Stephens et al. 2008). With the studies of unsupervised sessions, SinghGrewal and Stephens provided videos to assist (Singh-Grewal et al. 2007; Stephens et al. 2008), while Sousa had a session to teach parents as well (Sousa et al. 2012). The length of the intervention ranged from 5 weeks (Hernandez-Reif et al. 2001) to 1 year (Bao and Jin 2015), with the number of reported sessions ranging from 10 (Hernandez-Reif et al. 2001) to 72 (Tsang et al. 2009a, b, 2010, 2013), and five studies failed to report the total number of sessions.

\section{Effects of Intervention}

In assessing the effects of the intervention, there were two common themes for outcome measures: (1) psychological wellbeing and behavioral and (2) physical health and function (Table 3). Of the included manuscripts, seven reported positive improvements in participant's psychological wellbeing and behavior, while four showed neutral results and one reported worsening psychological health and wellbeing. Of the manuscripts reporting physical health and function, nine reported positive effects of the Tai Chi/Qigong intervention, with four reporting no net effect and zero reporting negative effects. Despite a common theme of outcome measures, the included manuscripts did not use the same tools (Table 4), except for two studies. The two studies with a common outcome measurement tool were studies evaluating the effects of Qigong in patient-specific populations, 
Table 3 Outcome measures of included intervention studies

\begin{tabular}{|c|c|c|c|c|}
\hline \multirow[t]{2}{*}{ Outcome measure } & \multicolumn{3}{|c|}{ Direction of results } & \multirow[t]{2}{*}{ Manuscripts } \\
\hline & + & \pm & - & \\
\hline \multicolumn{5}{|l|}{ Psychological wellbeing and behavioral effects } \\
\hline Behavior (participant self-assessment) & 1 & 0 & 0 & Tsang et al. (2009a, 2013) \\
\hline Behavior at home (parent assessment) & 0 & 1 & 0 & Witt et al. (2005) \\
\hline $\begin{array}{l}\text { Classroom behavior/performance (teacher } \\
\text { assessment) }\end{array}$ & 2 & 0 & 1 & Bao and Jin (2015), Hernandez-Reif et al. (2001) and Witt et al. (2005) \\
\hline Psychological health & 1 & 0 & 0 & Bao and Jin (2015) \\
\hline Quality of life & 1 & 1 & 0 & Singh-Grewal et al. (2007), Stephens et al. (2008) \\
\hline Stress & 2 & 2 & 0 & $\begin{array}{l}\text { Baron and Faubert (2005), Lee et al. (2013), Lozada et al. (2014) and } \\
\text { Sousa et al. (2012) }\end{array}$ \\
\hline \multicolumn{5}{|l|}{ Physical function and health effects } \\
\hline Disease-specific symptoms & 3 & 0 & 0 & Singh-Grewal et al. (2007), Stephens et al. (2008) and Zhang (1979) \\
\hline General health assessment & 3 & 0 & 0 & Singh-Grewal et al. (2007), Stephens et al. (2008) and Tsang et al. (2013) \\
\hline Physical activity & 2 & 1 & 0 & Chang et al. (2008), Stephens et al. (2008) and Tsang et al. (2013) \\
\hline Physical and cardiovascular fitness ${ }^{\mathrm{a}}$ & 1 & 1 & 0 & Singh-Grewal et al. (2007) and Tsang et al. (2009b, 2013) \\
\hline Weight management & 1 & 0 & 0 & Tsang et al. $(2009 b)$ \\
\hline
\end{tabular}

${ }^{\text {a}}$ Physical and cardiovascular fitness included aerobic and anaerobic capacity for cardiovascular fitness and muscle strength, power and endurance $+=$ positive conclusion; $\pm=$ conclusion neither positive nor negative conclusion; $-=$ negative conclusion

either fibromyalgia (Stephens et al. 2008) and juvenile idiopathic arthritis (Singh-Grewal et al. 2007), using the Children Health Assessment Questionnaire (CHAQ), and both studies reported improvement in Children Health Assessment Questionnaire scores. Positive reports were noted in the three studies that evaluate disease-specific conditions and general health through the Tai Chi or Qigong arm.

\section{Reporting and Methodological Quality}

Of the included manuscripts, seven manuscripts, which described four unique studies, were noted as being of high quality, and nine were of low quality (Table 5). A notable positive exhibited by the included manuscripts was the use of appropriate outcome measures, with these manuscripts providing evidence regarding the validity and reliability of the primary outcome measures. Moreover, although most studies were pilot and/or feasibility studies or single-arm trials, many manuscripts noted possible biases that may be present in the work. Finally, a considerable strength in the manuscripts reviewed was that all but two manuscripts provided a detailed description of a typical Tai Chi/Qigong session (Chang et al. 2011; Sousa et al. 2012).

Of the manuscripts of low quality, several studies did not identify confounding factors that may have had an impact on participant outcomes through the intervention period, such as age of the child, gender or disease status. For example, Lozada et al. (2014) included participants in 2nd (mean 7 years old) and 3rd (mean 8 years old) grade, without accounting for this age difference or development.
Although a 1 year age or grade may appear a small difference, at the primary school level this 1-year, one-grade difference between participants can affect executive function, inhibitory control and behavior problem symptomatology (Riggs et al. 2004), which was not accounted for in their work. Additionally, several manuscripts either did not explicitly report the percent completion or retention rates of the study or the study follow-up completion rate was less than $85 \%$ of the initial sample.

\section{Discussion}

The requisite high levels of concentrations that Tai Chi and Qigong necessitate for the sustained focus, precision in movement and strictness of action may not appeal to youth as it does to adults, which may affect efficacy of the intervention. Therefore, the aim of this review was to investigate studies of Tai Chi and Qigong in youth population as well as to synthesis outcomes and assess the methodological and reporting quality of these interventions. Overall, this systematic review showed that Tai Chi or Qigong may be an effective intervention to improve physical health and function, while there is insufficient evidence to evaluate its effect on psychological wellbeing and behavior. Further, results of this systematic review also reveal vast differences between these studies concerning the type, dose and duration of the Tai Chi/Qigong as well as the outcome measures studies. Lastly, the included studies show moderate methodological quality in terms of assessing the effects of Tai Chi or Qigong 


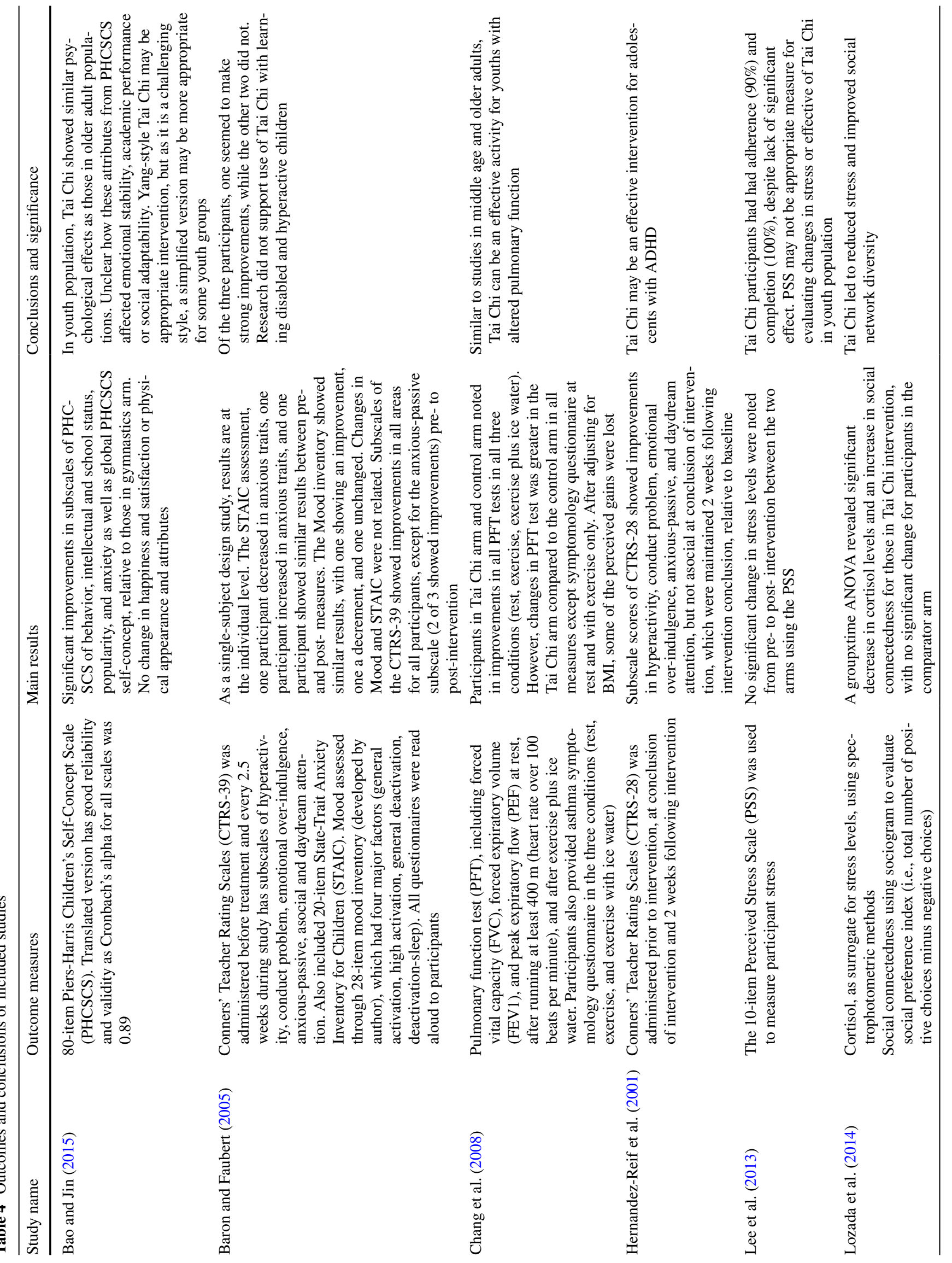




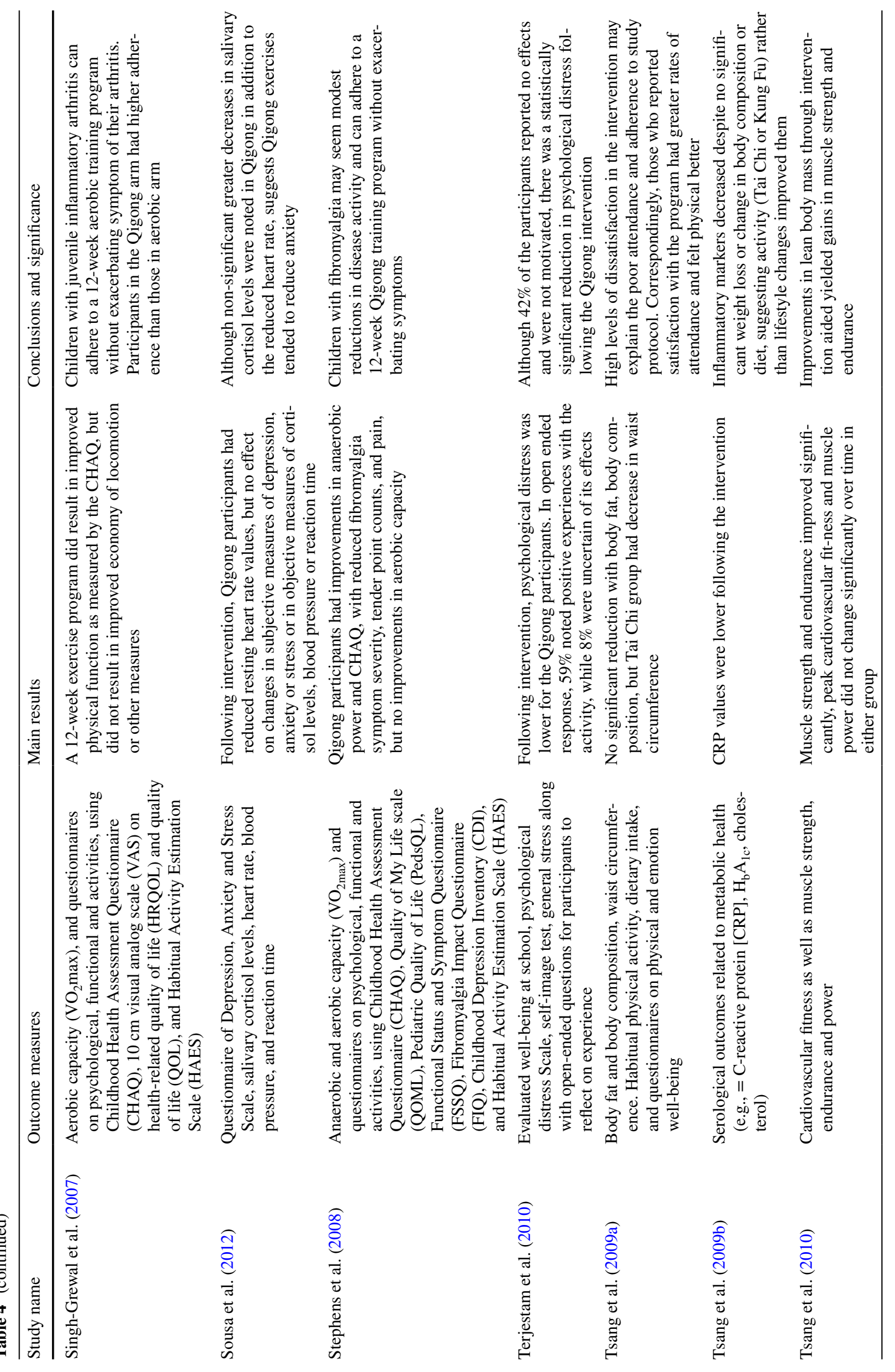




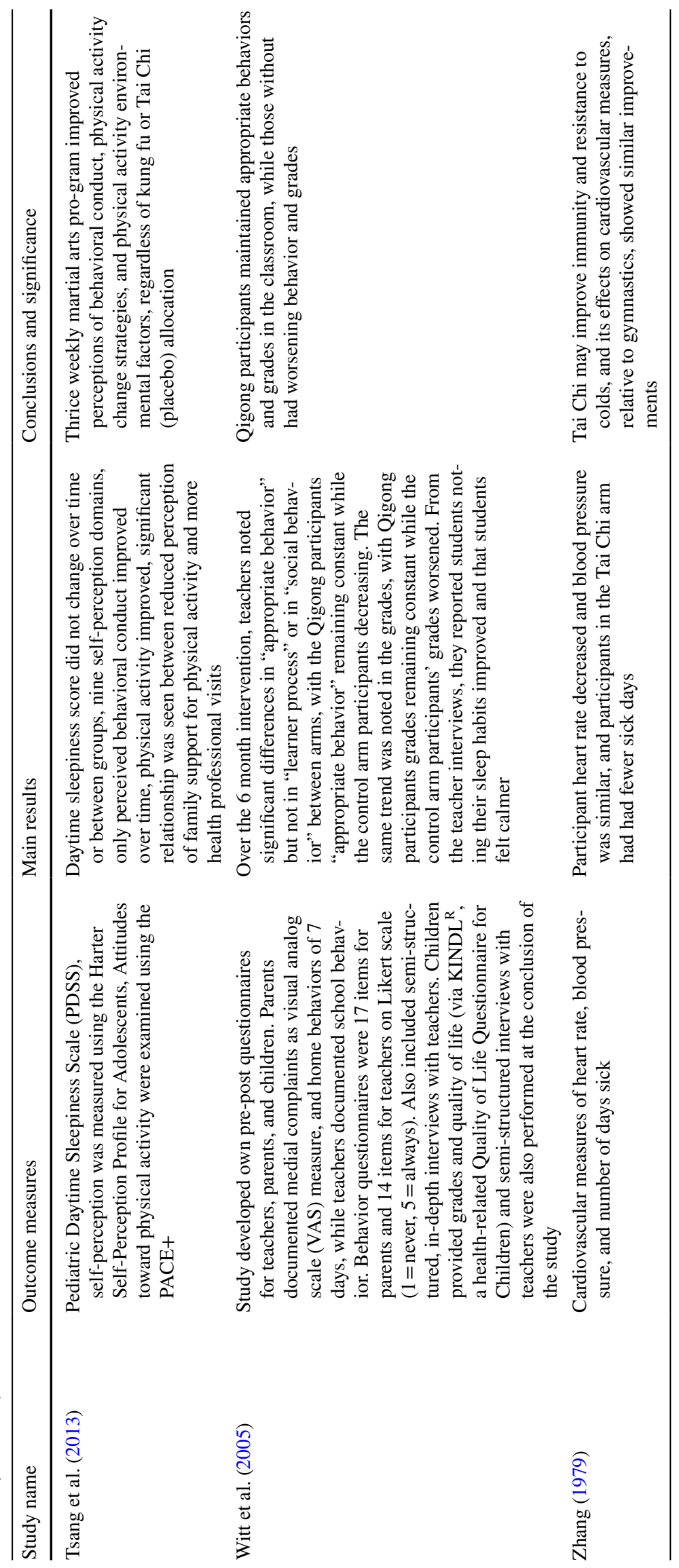




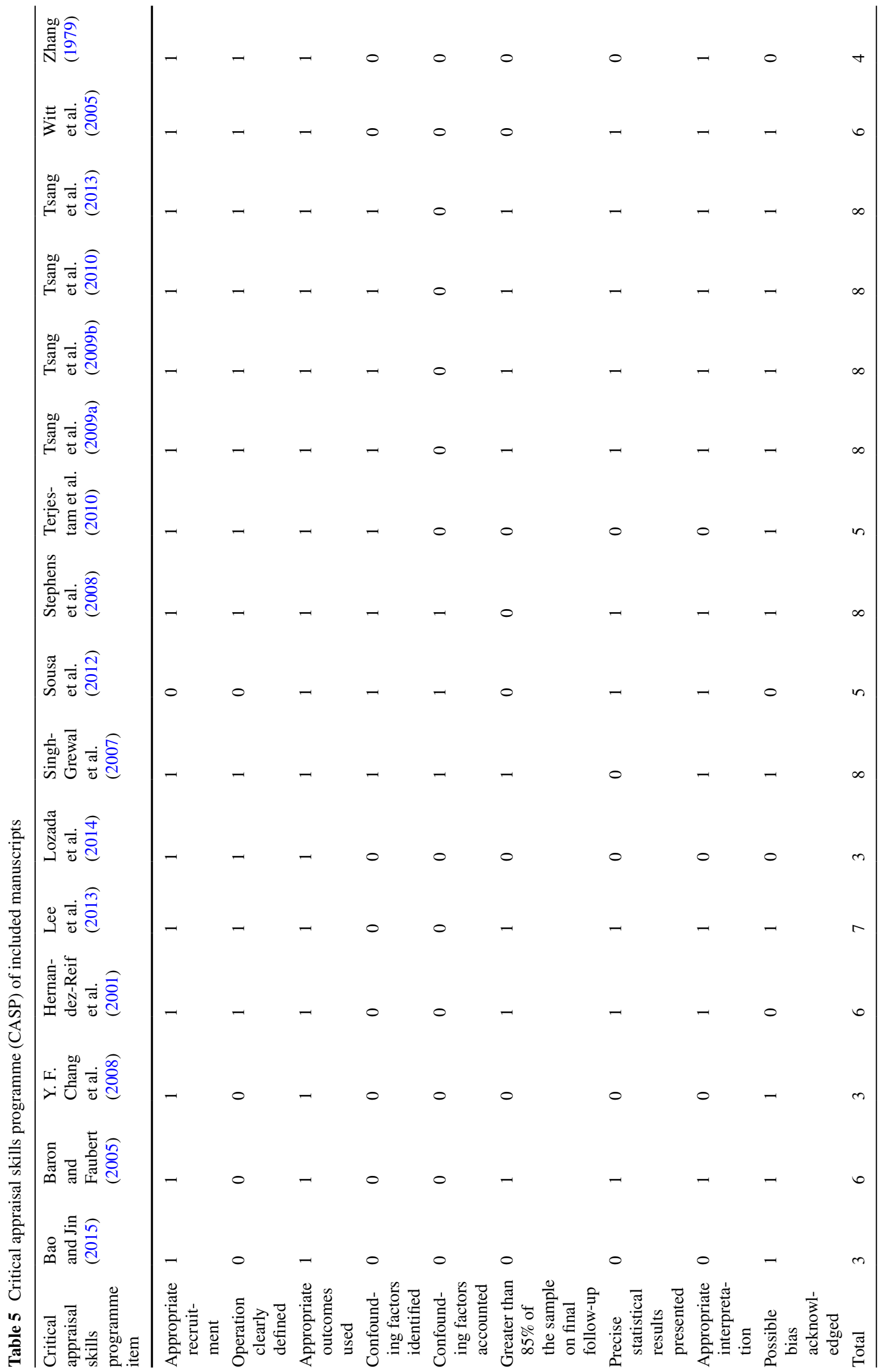


in a youth population, with notable reporting in the included studies lacking to effectively address confounders or biases in the study, suitably use statistical methods and reporting outcomes, and appropriately provide completion rates. The implications of this work suggest that although there may be physical health benefits of Tai Chi or Qigong, there is a strong need to properly evaluate the effects of said intervention in a robust manner as well as to further evaluate if there are psychological wellbeing or behavior effects.

Supporters of Tai Chi and Qigong report that it enhances flexibility, increases strength and improves balance (Taylor-Piliae et al. 2006). However, in youth populations, only strength has been fully evaluated, with studies suggest Tai Chi and Qigong can improve muscular strength (Tsang et al. 2010), endurance (Tsang et al. 2010), and anaerobic power (Sousa et al. 2012), which suggests there is a similarity between the physical benefits in younger and older populations.

From the perspective of child and adolescent developmental is the idea of neural maturation, which is the ageappropriate ability to control force in a given task (Kellis and Hatzitaki 2012). The ability to develop neural maturation through multi-joint movements, such as via Tai Chi or Qigong, may lead to greater efficiency in movement and greater likelihood of a healthy body weight trajectory (Lopes et al. 2012). As Tai Chi or Qigong practice involves controlled movements and changes of body position in different directions, it may be an appropriate intervention for improving the balance and coordination necessary for skill development in young children (Cordo and Gurfinkel 2004). Although balance is not typically an outcome measure in youth populations, its role along with body and hand-eye coordination, may be a fitting measure for youth populations (CôTé et al. 2009) as it is a foundation for developing the motor ability for complex movements (Cordo and Gurfinkel 2004). Gaining body awareness and motor control as well as improving and refining proprioception, which are constructs associated with motor proficiency, may yield higher physical activity levels and lower risk of obesity (Wrotniak et al. 2006). These results would suggest that improving these constructs (e.g., motor control, proprioception) through Tai Chi or Qigong practice at a young age may lead to benefits over time.

With the assumption Tai Chi or Qigong has a benefit on physical health and function, the potential mechanisms of action may be important. In older adults, regular Tai chi and Qigong have been hypothesized improvements in balance are through gains in trunk strength and trunk mobility (Wolf et al. 1996), which is often thought of as improvement in "core" strength and mobility, while others have suggested it is through gains in lower extremity strength, mobility, and peripheral somatosensation (Cavegn and Riskowski 2015). With respect to youth population, there is no evidence regarding changes in core strength or mobility or in lower extremity mobility or peripheral sensation. Future research could build from the understanding of studies in adult population to evaluate if there are changes in neuromuscular control, core strength and peripheral sensation that arise through Tai Chi or Qigong participation.

Moreover, of the studies that evaluated the physical benefits of Tai Chi or Qigong, all but one (Zhang 1979) evaluated it through a randomized controlled trial design, providing strong evidence of an effect, and were at least 12 weeks in duration. Though it is hard to determine exact number of hours in the included studies given some of the poor reporting, these results that Tai Chi or Qigong improves physical health and function may align with research in older adult populations that suggest physical improvement is most evident after $40 \mathrm{~h}$ of Tai Chi practice (Wu 2002), and an intervention of 12 weeks may provide sufficient training to improve physical fitness in young children (Strong et al. 2005). However, the question of whether Tai Chi or Qigong is superior to other therapeutic exercise intervention is currently unanswered and is a topic for further investigation. Nonetheless, when included studies compared Tai Chi or Qigong to other physical activities that would generally deemed to more physically active (i.e., martial arts (Tsang et al. 2010) or vigorous aerobic training (Stephens et al. 2008)), Tai Chi/Qigong showed similar physical gains (e.g., strength, endurance) with few adverse events (none in the two studies that reported it), suggesting it may be an appropriate intervention for children and adolescents to improve physical health.

Understanding the effects of Tai Chi or Qigong on mental health and psychosocial wellbeing in youth is more challenging. In older adult populations, a systematic review and meta-analysis showed Tai Chi and Qigong positively improved participants' psychosocial quality of life (Wang et al. 2014), with the caveat that methodology quality of the included studies was not consistently strong. For this review, psychological wellbeing and behavior was the overall domain of interest, which included measures of behavior with perspectives from the participant (number of studies $=1)$, parent/guardian $(\mathrm{N}=1)$ and classroom teacher $(\mathrm{N}=3)$, psychological health $(\mathrm{N}=1)$, quality of life $(\mathrm{N}=2)$ and stress $(\mathrm{N}=2)$. In these areas of psychological wellbeing and behavior there were no consistent results across the studies, and at best, the results of the work appear that Tai Chi or Qigong may improve or may have no effect on this domain.

In measuring behavior, none of the studies attempted to triangulate the results across the different perspectives, and only one study included two perspectives (parent and teacher) (Witt et al. 2005), which showed parents reporting no change in behavior and teachers noting an improvement. Guidelines for measuring children and adolescent behavior and wellbeing have suggested children and the proxy (e.g., 
parents, teachers) should be provided with parallels forms in order to gain a potentially more reliable source of information (Wallander et al. 2001). Further, one study noted that with Tai Chi students perceived the practice to lead to a greater connection with peers and increased social circles (Lozada et al. 2014), suggesting the may be an shift at the time or the way in which adolescents move from parental support to peer support (Helsen et al. 2000). However, the mechanism by which these changes in behavior and movement from parental to peer support in adolescence through Tai Chi or Qigong need to be further investigated to determine if there is a true effect and the underpinnings of how they occur.

With respect to quality of life, despite guidelines that suggest studies should include both objective and subjective approaches to measuring quality of life (Wallander et al. 2001), only one study included an objective measure alongside the subjective measure (Sousa et al. 2012), and its results showed similar outcomes with the both the objective and subjective measures of stress showing no change over the course of the Qigong intervention. As such, future studies that include psychological wellbeing and behaviors should include similar instruments to the child or adolescent and a proxy, as well as include subjective and objective measures where possible.

Although we noted that Tai Chi and Qigong may have positive physical health and mobility benefits for youth population, the results of this review should be couched with respect to the study's strengths and limitations. First, the selection criteria excluded unpublished or non-peer reviewed material such as university thesis, protocols, guidelines, or grey literature. Although, this was undertaken to ensure that the material reviewed had been through a peer-review process to attempt to maintain quality, it may permit the potential for publication bias to influence this review's results (Rothstein et al. 2005). Further, the search strategy used was based on a computer search, which may omit some articles and limit the scope of the review (Colville-Stewart 2002). To address this, hand searchers of the reference lists of the included manuscripts were evaluated to determine if other manuscripts were missed. Of the 16 included manuscripts, there were three studies of Tai Chi that were not included from the initial search (keyword stage); none of these studies met the inclusion criteria. Further, although there was no language limits applied, the keyword search terms used were English language words. As such only manuscripts with a title and/or abstract written in the English would have been extracted through the process. Nonetheless, the review did include a study written in Chinese, so it was possible to included non-English language studies. Searches in databases using keywords in other languages may yield different results. With respect to the manuscripts, the included studies varied in population, other than being in a youth population, and outcomes assessed. Due to this heterogeneity and nature of the study design, a statistically assessed meta-analysis would not have been appropriate (Egger et al. 2001; Polgar and Thomas 2000), but the semi-qualitative approach taken in synthesizing the results provides a level of generalizability of the review's findings (Polgar and Thomas 2000). Similarly, a formal assessment of publication bias was not conducted given the lack of a formal synthesis of results. Nonetheless, the strength of this study was its inclusion to all studies of Tai Chi and Qigong in youth population. As such, this review provides a foundation for Tai Chi or Qigong interventions in youth populations, and it may assist in determining appropriate outcome measures and design characteristics for future studies.

To build from these included studies and systematic review of the work, future trials of Tai Chi or Qigong in youth populations should adhere to accepted standards of trial methodology and trial reporting in order to more fully evaluate the effect of the applied intervention. Studies should present a more detailed report of the Tai Chi or Qigong instructor, a clear explanation of the frequency and duration of the intervention, and adequate use statistical testing and reporting. Additionally, despite the challenges of blinding participant by treatment in physical activity intervention, assessor blinding and allocation concealment are important for reducing bias. With respect to outcome measures, studies should evaluate the effect of Tai Chi or Qigong on balance and coordination as well as on participant behavior as assessed by multiple sources, including participant selfassessing, if appropriate, along with parents/guardians, and/ or teachers and triangulating results to determine if there is truly an effect.

\section{Conclusion}

There are encouraging results suggesting Tai Chi or Qigong may be effective for improving physical health and function in for children and adolescents, while its effect on psychological health and behaviors needs further evaluation in order to understand if there is an effect. In this review, the main benefits noted with Tai Chi or Qigong were with respect to disease-specific symptoms and general health; however, these studies also had focused populations with specific physical health conditions. As such, the effect of Tai Chi or Qigong on physical health in the general population is unknown. Further, there were four unique studies that evaluated participant's behavior, with only one study evaluating behavior from multiple perspectives, which in this case two perspectives: parent and classroom teacher. Future studies, if they are to address the adolescent's behavior should evaluate behavior from more than one perspective. Similarly only one study noted a potential shift in participants developing 
their social circle through the Tai Chi practice, suggesting a greater perceived level of support from their peers, but these results should be evidenced by further studies. Moreover, owing to the number of eligible randomized controlled trials or controlled trials was the often poor quality and high likelihood of bias in the studies, suggesting As such, future studies should be more rigorous in their design and reporting. Specific areas to address study design and reporting include a detail account of the Tai Chi or Qigong session, including a description of the instructor background, and identifying and accounting for confounding factors, such as differences in participant development stage or age (if there is a range) or participant bias to the activity to address placebo effect of the intervention. In short, while there are few studies evaluating Tai Chi or Qigong in youth populations, there are known benefits for physical health in both general and disease-specific population, while its effects on psychological health and behavior effects as well as adolescent development need further research.

Acknowledgements The authors would like to thank Xinhua Shu, $\mathrm{PhD}$ for his assistance throughout the systematic review.

Author Contributions JLR conceived of the study, and JLR and AR participated in its design and coordination and drafted the manuscript All authors read and approved the final manuscript.

\section{Compliance with Ethical Standards}

Conflict of interest The authors report no conflicts of interest.

Open Access This article is distributed under the terms of the Creative Commons Attribution 4.0 International License (http://creativecommons.org/licenses/by/4.0/), which permits unrestricted use, distribution, and reproduction in any medium, provided you give appropriate credit to the original author(s) and the source, provide a link to the Creative Commons license, and indicate if changes were made.

\section{References}

Bao, X., \& Jin, K. (2015). The beneficial effect of Tai Chi on self-concept in adolescents. International Journal of Psychology, 50(2), 101-105. doi:10.1002/ijop.12066.

Barnes, P. M., Bloom, B., \& Nahin, R. L. (2008). Complementary and alternative medicine use among adults and children: United States, 2007: US Department of Health and Human Services, Centers for Disease Control and Prevention, National Center for Health Statistics Hyattsville, MD.

Baron, L. J., \& Faubert, C. (2005). The role of Tai Chi Chuan in reducing state anxiety and enhancing mood of children with special needs. Journal of Bodywork and Movement Therapies, 9(2), $120-133$.

Ben-Arye, E., Bar-Sela, G., Frenkel, M., Kuten, A., \& Hermoni, D. (2006). Is a biopsychosocial-spiritual approach relevant to cancer treatment? A study of patients and oncology staff members on issues of complementary medicine and spirituality. Supportive Care in Cancer, 14(2), 147-152. doi:10.1007/s00520-005-0866-8.
Cavegn, E. I., \& Riskowski, J. L. (2015). The effects of Tai Chi on peripheral somatosensation, balance, and fitness in hispanic older adults with type 2 diabetes: A pilot and feasibility study. Evidence-Based Complementary and Alternative Medicine, 2015, 767213. doi:10.1155/2015/767213.

Chacko, S. A., Wee, C. C., Davis, R. B., \& Yeh, G. a. Y. (2014). Use of Meditation and Breathing Exercises in Mind-Body Exercise in the U.S. The Journal of Alternative and Complementary Medicine, 20(5), A121-A121. doi:10.1089/acm.2014.5321.abstract.

Chang, J. Y., Tsai, P. F., Beck, C., Hagen, J. L., Huff, D. C., Anand, K. J.,. .. Beuscher, L. (2011). The effect of tai chi on cognition in elders with cognitive impairment. Medsurg Nursing, 20(2), 63-69 (quiz 70).

Chang, Y. F., Yang, Y. H., Chen, C. C., \& Chiang, B. L. (2008). Tai Chi Chuan training improves the pulmonary function of asthmatic children. Journal of microbiology, immunology, and infection, 41(1), 88-95.

Chen, Z., Meng, Z., Milbury, K., Bei, W., Zhang, Y., Thornton, B., Cohen, L. (2013). Qigong improves quality of life in women undergoing radiotherapy for breast cancer: results of a randomized controlled trial. Cancer, 119(9). doi:10.1002/cncr.27904.

Clarke, T. C., Black, L. I., Stussman, B. J., Barnes, P. M., \& Nahin, R. L. (2015). Trends in the use of complementary health approaches among adults: United States, 2002-2012. National Health Statistics Reports, 79, 1.

Colville-Stewart, S. (2002). How to do a literature search. In M. Tarling \& L. Croft (Eds.), The essential researcher's handbook. For nurses and healthcare professionals (2nd edn., pp. 35-53). London: Baillie`re Tindall.

Cordo, P. J., \& Gurfinkel, V. S. (2004). Motor coordination can be fully understood only by studying complex movements. Progress in Brain Research, 143, 29-38. doi:10.1016/ s0079-6123(03)43003-3.

CôTé, J., Lidor, R., \& Hackfort, D. (2009). ISSP position stand: To sample or to specialize? Seven postulates about youth sport activities that lead to continued participation and elite performance. International Journal of Sport and Exercise Psychology, 7(1), $7-17$.

Critical Skills Appraisal Programme-CASP (2007) (2007). Retrieved June 11, 2015, from http://www.phru.nhs.uk/casp/critical_ appraisal_tools.htm.

Egger, M., Smith Davey, G., \& Schneider, M. (2001). Systematic reviews of observational studies. In M. Egger, G. Davey Smith \& D. G. Altman (Eds.), Systematic Reviews in Health Care (pp. 211228). London: BMJ Books.

Elkins, G., Fisher, W., \& Johnson, A. (2010). Mind-Body Therapies in Integrative Oncology. Current Treatment Options in Oncology, 11(3), 128-140. doi:10.1007/s11864-010-0129-x.

Evans, D. (2002). Systematic reviews of interpretive research: Interpretive data synthesis of processed data. The Australian Journal of Advanced Nursing, 20(2), 22-26.

Faggion, C. M. Jr. (2016). The rationale for rating risk of bias should be fully reported. Journal of Clinical Epidemiology, 76, 238. doi:10.1016/j.jclinepi.2016.03.007.

Fuzhong, L., Peter, H., Edward, M., Duncan, T. E., Duncan, S. C., Nigel, C., \& Fisher, K. J. (2001). An evaluation of the effects of Tai Chi exercise on physical function among older persons: A randomized controlled trial. Annals of Behavioral Medicine, 23(2), 139-146. doi:10.1207/S15324796ABM2302_9.

Goon, J. A., Aini, A. H. N., Musalmah, M., Anum, M. Y. Y., Nazaimoon, W. M. W., \& Ngah, W. Z. W. (2009). Effect of Tai Chi exercise on DNA damage, antioxidant enzymes, and oxidative stress in middle-age adults. Journal of Physical Activity and Health, 6(1), 43-54.

Gryffin, P. A., Chen, W. C., Chaney, B. H., Dodd, V. J., \& Roberts, B. (2015). Facilitators and Barriers to Tai Chi in an Older Adult 
Community: A Theory-Driven Approach. American Journal of Health Education, 46(2), 109-118. doi:10.1080/19325037.2014 .999964.

Gueguen, J., Piot, M.-A., Orri, M., Gutierre, A., Le Moan, J., Berthoz, S., \& Godart, N. (2017). Group Qigong for Adolescent Inpatients with Anorexia Nervosa: Incentives and Barriers. PLoS One, 12(2), e0170885. doi:10.1371/journal.pone.0170885.

Hackney, M. E., \& Earhart, G. M. (2008). Tai Chi improves balance and mobility in people with Parkinson disease. Gait and Posture, 28(3), 456-460.

Harris, P. E., Cooper, K. L., Relton, C., \& Thomas, K. J. (2012). Prevalence of complementary and alternative medicine (CAM) use by the general population: a systematic review and update. International Journal of Clinical Practice, 66(10), 924-939. doi:10.1111/j.1742-1241.2012.02945.x.

Helsen, M., Vollebergh, W., \& Meeus, W. (2000). Social Support from Parents and Friends and Emotional Problems in Adolescence. Journal of Youth and Adolescence, 29(3), 319-335. doi:10.102 3/A:1005147708827.

Hernandez-Reif, M., Field, T. M., \& Thimas, E. (2001). Attention deficit hyperactivity disorder: benefits from Tai Chi. Journal of Bodywork and Movement Therapies, 5(2), 120-123.

Jahnke, R., Larkey, L., Rogers, C., Etnier, J., \& Lin, F. (2010). A Comprehensive Review of Health Benefits of Qigong and Tai Chi. American Journal of Health Promotion, 24(6), e1-e25. doi:10.4278/ajhp.081013-LIT-248.

Kang, J. W., Lee, M. S., Posadzki, P., \& Ernst, E. (2011). T'ai chi for the treatment of osteoarthritis: a systematic review and metaanalysis. BMJ Open, 1(1), e000035.

Kellis, E., \& Hatzitaki, V. (2012). 3 Development of Neuromuscular Coordination with Implications in Motor Control. In Paediatric biomechanics and motor control. Theory and application (pp. 50-70). New York, NY: Routledge, Taylor \& Francis Group.

Lan, C., Lai, J. S., Chen, S. Y., \& Wong, M. K. (1998). 12-month Tai Chi training in the elderly: its effect on health fitness. Medicine and Science in Sports and Exercise, 30(3), 345-351.

Larkey, L., Jahnke, R., Etnier, J., \& Gonzalez, J. (2009). Meditative movement as a category of exercise: implications for research. Journal of Physical Activity \& Health, 6(2), 230-238.

Lee, L. Y. K., Chong, Y. L., Li, N. Y., Li, M. C., Lin, L. N., Wong, L. Y., \& Man, S. Y. (2013). Feasibility and effectiveness of a Chen Tai Chi programme for stress reduction in junior secondary school students. Stress and Health: Journal of the International Society for the Investigation of Stress, 29(2), 117-124. doi:10.1002/ smi.2435.

Levac, D., Colquhoun, H., \& O’Brien, K. K. (2010). Scoping studies: Advancing the methodology. Implementation Science, 5(1), 1. doi:10.1186/1748-5908-5-69.

Liu, H., \& Frank, A. (2010). Tai Chi as a balance improvement exercise for older adults: a systematic review. Journal of Geriatric Physical Therapy, 33(3), 103-109. doi:10.1097/JPT.0b013e3181eda1c4.

Lopes, V. P., Maia, J. A. R., Rodrigues, L. P., \& Malina, R. (2012). Motor coordination, physical activity and fitness as predictors of longitudinal change in adiposity during childhood. European Jounral of Sport Science, 12(4), 384-391. doi:10.1080/1746139 1.2011.566368.

Lozada, M., Carro, N., D’Adamo, P., \& Barclay, C. (2014). Stress management in children: a pilot study in 7-9 year olds. Journal of Developmental and Behavioral Pediatrics, 35(2), 144-147. doi:10.1097/dbp.0000000000000026.

Moher, D., Liberati, A., Tetzlaff, J., Altman, D. G., \& Group, T. P. (2009). Preferred reporting items for systematic reviews and metaanalyses: The PRISMA statement. PLoS Medicine, 6(7), 6.

Niu, R., He, R., Luo, B. L., \& Hu, C. (2014). The effect of tai chi on chronic obstructive pulmonary disease: a pilot randomised study of lung function, exercise capacity and diaphragm strength.
Heart, Lung and Circulation, 23(4), 347-352. doi:10.1016/j. hlc.2013.10.057.

Polgar, S., \& Thomas, S. A. (2000). Introduction to research in the health sciences (4th edn.). London.

Riggs, N. R., Blair, C. B., \& Greenberg, M. T. (2004). Concurrent and 2-year longitudinal relations between executive function and the behavior of 1st and 2nd grade children. Child Neuropsychology, 9(4), 267-276.

Rothstein, H. R., Sutton, A. J., \& Borenstein, M. (2005). Publication bias in meta-analysis. In H. R. Rothstein, A. J. Sutton \& M. Borenstein (Eds.), Publication bias in meta-analysis. Prevention, assessment and adjustment (pp. 1-7). (Chichester: Wiley).

Sanderson, S., Tatt, I. D., \& Higgins, J. P. (2007). Tools for assessing quality and susceptibility to bias in observational studies in epidemiology: a systematic review and annotated bibliography. International Journal of Epidemiology, 36(3), 666-676.

Singh-Grewal, D., Schneiderman-Walker, J., Wright, V., Bar-Or, O., Beyene, J., Selvadurai, H., \& Feldman, B. M. (2007). The effects of vigorous exercise training on physical function in children with arthritis: A randomized, controlled, SINGLE-BLINDED trial. Arthritis Care and Research, 57(7), 1202-1210. doi:10.1002/ art.23008.

Slavin, R. E. (1995). Best evidence synthesis: an intelligent alternative to meta-analysis. Journal of Clinical Epidemiology, 48(1), 9-18.

Sousa, C. M., Goncalves, M., Machado, J., Efferth, T., Greten, T., Froeschen, P., \& Greten, H. J. (2012). Effects of qigong on performance-related anxiety and physiological stress functions in transverse flute music schoolchildren: a feasibility study. Zhong Xi Yi Jie He Хиe Bao, 10(8), 858-865.

Stephens, S., Feldman, B. M., Bradley, N., Schneiderman, J., Wright, V., Singh-Grewal, D., \& Tse, S. M. (2008). Feasibility and effectiveness of an aerobic exercise program in children with fibromyalgia: results of a randomized controlled pilot trial. Arthritis and Rrheumatism, 59(10), 1399-1406. doi:10.1002/art.24115.

Straus, S. (2000). Expanding horizons of healthcare, 5-year strategic plan 2001-2005. National Centre for Complementary and Alternative Medicine.

Strong, W. B., Malina, R. M., Blimkie, C. J. R., Daniels, S. R., Dishman, R. K., Gutin, B., \& Pivarnik, J. M. (2005). Evidence based physical activity for school-age youth. The Journal of Pediatrics, 146(6), 732-737.

Taylor-Piliae, R. E., Haskell, W. L., Stotts, N. A., \& Froelicher, E. S. (2006). Improvement in balance, strength, and flexibility after 12 weeks of Tai chi exercise in ethnic Chinese adults with cardiovascular disease risk factors. Alternative Therapies in Health and Medicine, 12(2), 50-58.

Terjestam, Y., Jouper, J., \& Johansson, C. (2010). Effects of scheduled qigong exercise on pupils' well-being, self-image, distress, and stress. Journal of Alternative and Complementary Medicine, 16(9), 939-944. doi:10.1089/acm.2009.0405.

Thornton, E. W., Sykes, K. S., \& Tang, W. K. (2004). Health benefits of Tai Chi exercise: improved balance and blood pressure in middleaged women. Health Promotion International, 19(1), 33-38.

Tsang, T. W., Kohn, M., Chow, C. M., \& Singh, M. F. (2009a). A randomised placebo-exercise controlled trial of Kung Fu training for improvements in body composition in overweight/obese adolescents: the "Martial Fitness" study. Journal of Sports Science and Medicine, 8(1), 97-106.

Tsang, T. W., Kohn, M., Chow, C. M., \& Singh, M. F. (2009b). A randomized controlled trial of Kung Fu training for metabolic health in overweight/obese adolescents: the "martial fitness" study. Journal of Pediatric Endocrinology and Metabolism, 22(7), 595-607. http://onlinelibrary.wiley.com/o/cochrane/clcentral/articles/953/ CN-00721953/frame.html.

Tsang, T. W., Kohn, M. R., Chow, C. M., \& Fiatarone Singh, M. A. (2010). Kung fu training improves physical fitness measures in 
overweight/obese adolescents: the "martial fitness" study. Journal of Obesity. doi:10.1155/2010/672751.

Tsang, T. W., Kohn, M. R., Chow, C. M., \& Singh, M. F. (2013). Selfperception and attitude toward physical activity in overweight/ obese adolescents: the "martial fitness" study. Research in Sports Medicine, 21(1), 37-51. doi:10.1080/15438627.2012.738444.

Uhlig, T., Fongen, C., Steen, E., Christie, A., \& Ødegård, S. (2010). Exploring Tai Chi in rheumatoid arthritis: a quantitative and qualitative study. BMC Musculoskeletal Disorders, 11(1), 43. doi:10.1186/1471-2474-11-43.

Wall, R. B. (2005). Tai Chi and mindfulness-based stress reduction in a Boston Public Middle School. Journal of Pediatric Health Care, 19(4), 230-237. doi:10.1016/j.pedhc.2005.02.006.

Wallander, J. L., Schmitt, M., \& Koot, H. M. (2001). Quality of life measurement in children and adolescents: issues, instruments, and applications. Journal of Clinical Psychology, 57(4), 571-585.

Wang, C., Bannuru, R., Ramel, J., Kupelnick, B., Scott, T., \& Schmid, C. H. (2010). Tai Chi on psychological well-being: systematic review and meta-analysis. BMC Complementary and Alternative Medicine, 10, 23-23. doi:10.1186/1472-6882-10-23.

Wang, C., Collet, J. P., \& Lau, J. (2004). The effect of Tai Chi on health outcomes in patients with chronic conditions: a systematic review. Archives of Internal Medicine, 164(5), 493-501. doi:10.1001/ archinte.164.5.493.

Wang, C., Schmid, C. H., Hibberd, P. L., Kalish, R., Roubenoff, R., Rones, R., \& McAlindon, T. (2009). Tai Chi is effective in treating knee osteoarthritis: a randomized controlled trial. Arthritis Care and Research (Hoboken), 61(11), 1545-1553.

Wang, F., Lee, E. K., Wu, T., Benson, H., Fricchione, G., Wang, W., \& Yeung, A. S. (2014). The effects of tai chi on depression, anxiety, and psychological well-being: a systematic review and metaanalysis. International Journal of Behavioral Medicine, 21(4), 605-617. doi:10.1007/s12529-013-9351-9.

Witt, C., Becker, M., Bandelin, K., Soellner, R., \& Willich, S. N. (2005). Qigong for schoolchildren: a pilot study. Journal of Alternative and Complementary Medicine, 11(1), 41-47. doi:10.1089/ acm.2005.11.41.
Wolf, S. L., Barnhart, H. X., Kutner, N. G., McNeely, E., Coogler, C., \& Xu, T. (1996). Reducing frailty and falls in older persons: an investigation of Tai Chi and computerized balance training. Journal of the American Geriatrics Society, 44(5), 489-497.

Wolfson, L., Whipple, R., Derby, C., Judge, J., King, M., Amerman, P., \& Smyers, D. (1996). Balance and strength training in older adults: intervention gains and Tai Chi maintenance. Journal of the American Geriatrics Society, 44(5), 498-506.

Wrotniak, B. H., Epstein, L. H., Dorn, J. M., Jones, K. E., \& Kondilis, V. A. (2006). The relationship between motor proficiency and physical activity in children. Pediatrics, 118(6), e1758-1765. doi:10.1542/peds.2006-0742.

Wu, G. (2002). Evaluation of the effectiveness of Tai Chi for improving balance and preventing falls in the older population-a review. Journal of the American Geriatrics Society, 50(4), 746-754.

Yan, J.-H., Gu, W.-J., Sun, J., Zhang, W.-X., Li, B.-W., \& Pan, L. (2013). Efficacy of Tai Chi on pain, stiffness and function in patients with osteoarthritis: a meta-analysis. PLoS One, 8(4), e61672. doi:10.1371/journal.pone.0061672.

Yang, G.-Y., Wang, L.-Q., Ren, J., Zhang, Y., Li, M.-L., Zhu, Y.-T., \& Liu, J.-P. (2015). Evidence base of clinical studies on Tai Chi: A bibliometric analysis. PLoS One, 10(3), e0120655. doi:10.1371/ journal.pone.0120655.

Ye, J., Cai, S., Zhong, W., Cai, S., \& Zheng, Q. (2014). Effects of Tai Chi for patients with knee osteoarthritis: A systematic review. Journal of Physical Therapy Science, 26(7), 1133-1137. doi:10.1589/jpts.26.1133.

Zhang, G. D. (1979). [Effect of simplified Tai-Ji boxing and playing in water on the health of children with abnormal cardiovascular system (author's transl)]. Zhonghua yu fang yi xue za zhi [Chinese journal of preventive medicine], 13(3), 136-138.

Zhuo, D., Shephard, R. J., Plyley, M. J., \& Davis, G. M. (1984). Cardiorespiratory and metabolic responses during Tai Chi Chuan exercise. Canadian Journal of Applied Sport Sciences, 9(1), 7-10. 